\title{
Research on High-Speed Railway Freight Train Organization Method considering Different Transportation Product Demands
}

\author{
Xueqiao Yu, ${ }^{1}$ Lingyun Zhou ${ }^{(D)},{ }^{1}$ Mingkun Huo, ${ }^{2}$ and Xiao $\mathrm{Yu}^{3}$ \\ ${ }^{1}$ Transportation and Economics Research Institute, China Academy of Railway Sciences Corporation Limited, \\ Beijing 100081, China \\ ${ }^{2}$ CCCC Intelligence Transportation Company Limited, Tianjin 300210, China \\ ${ }^{3}$ Signal and Communication Research Institute, China Academy of Railway Sciences Corporation Limited, Beijing 100081, China \\ Correspondence should be addressed to Lingyun Zhou; zhoulingyun@rails.cn
}

Received 10 February 2021; Revised 15 June 2021; Accepted 27 June 2021; Published 19 July 2021

Academic Editor: Eugenio Roanes Lozano

Copyright (C) 2021 Xueqiao Yu et al. This is an open access article distributed under the Creative Commons Attribution License, which permits unrestricted use, distribution, and reproduction in any medium, provided the original work is properly cited.

\begin{abstract}
The sustained and stable growth of China's market economy promotes the continuous improvement of transportation demand and puts forward higher requirements for transportation products. At present, as a new product, high-speed rail freight has played an important role in cold chain logistics and high-value-added transportation, which is of great significance to effectively reduce the pollution caused by road transportation and further promote the sustainable development of economy and logistics industry. In the previous study, we studied the organization optimization method of high-speed rail freight train operation scheme and the compilation method of high-speed rail freight train diagram based on the overall demand of freight transportation but did not consider the influence of different high-speed rail freight transportation products on the organization scheme. Therefore, in order to ensure the sustainable development of high-speed rail freight business, based on the demand of high-speed freight products (the freight product for short in the following text), this paper focuses on the optimization method of high-speed rail freight train organization scheme considering different demands. A two-stage freight demand analysis method considering customers' choice intention and actual supply capacity was designed, comprehensively considering the relationship between operation cost and income, and a model based on different products in order to meet the needs of large-scale case solving was established. In addition, a column generation algorithm was designed. The computational results are encouraging and demonstrate the effectiveness of the model and solution method.
\end{abstract}

\section{Introduction}

In recent years, with the sustained and stable development of China's economy, China's logistics industry has developed rapidly, and the demand for fast freight transportation has increased at a fast pace. In 2020, China's express business volume reached 83.4 billion pieces, and it has been the world's largest express delivery country for seven consecutive years, accounting for $55 \%$ of the total global express business volume. In recent five years, the compound growth rate of express business is $32 \%$. Especially in 2020, due to the impact of COVID-19, the express business still maintains a high-speed growth trend, making an important contribution to China's economic development and ensuring the safety and reliability of the global supply chain. The growth trend of China's express business volume and business income from 2008 to 2020 is shown in Figure 1.

At present, most of China's express delivery demand is domestic transportation demand between different places, which not only brings high profit space for the express delivery market but also puts forward increasing requirements and challenges for the effective supply of transportation mode to meet the demand. How to ensure reasonable utilization of transportation resources has become the key problem to meet the express delivery demand. Since its launch, the high-speed rail freight business has been growing at a fast pace with an average annual compound growth rate of more than $30 \%$. It is conservatively estimated 


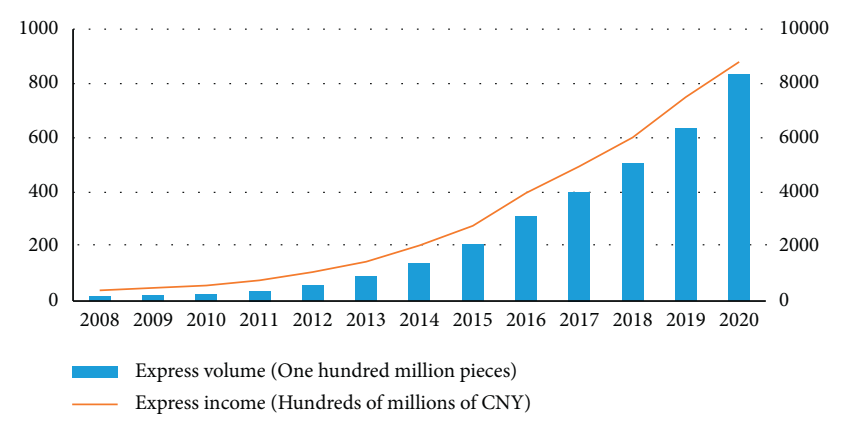

Figure 1: The volume and income of express delivery in 2008-2020.

that by 2035 , the scale of express delivery market will reach 270 billion pieces, of which the annual demand of high-end express delivery market in the range of $500-2500 \mathrm{~km}$ is about 11.5 billion pieces, and the daily demand of high-speed rail freight EMUs will reach 570 freight trains. Therefore, faced with the huge demand of high-speed rail freight market, how to improve the effective product supply has become the main problem to be solved.

In the previous two articles, we focused on the train organization plan [1] and the specific operation plan [2], that is, to optimize the train operation interval, stop mode, and operation time. However, with the rapid development of high-speed rail freight business, the transport market puts forward higher requirements for transport products, and the demand of different transport products varies greatly. Therefore, the overall freight demand of high-speed rail freight should not be taken as the basis for making a train operation plan, but the demand of different transport products should be calculated specifically, so as to make a better transport organization plan. On the basis of existing research, we focus on optimizing the design of cargo flow allocation model, achieving the accurate division of different cargo flows, and improving the effective supply level.

In order to fully meet the differentiated market demand, this paper designs the calculation method of demand sharing rate of the freight product, constructs a two-stage optimization method of high-speed rail freight train organization scheme considering the transportation demand of different products, and reveals the influence mechanism of transportation demand on transportation supply. The remainder of this paper is organized as follows: Section 2 introduces the related research review. In Section 3, the calculation method of freight flow sharing rate of high-speed railway is designed. Section 4 establishes the train candidate set generation model of a high-speed railway express train. In Section 5, an algorithm was designed to solve the model. Section 6 constructs a high-speed express train flow distribution model considering the different product demand. Finally, an example is used to generate the high-speed railway express train operation plan.

\section{Literature Review}

With regard to freight flow share rate, the essential problem is the analysis of the competition relationship of different transportation products. Researchers have done in-depth research on the competitiveness of the transportation market and built a mathematical model that can objectively describe the actual problems, so as to analyze the choice of transportation mode, career choice, and enterprise development decision-making in the transportation channel. In terms of passenger flow research, Talebian and Zou [3] proposed a three-stage game model to study the railway competitiveness in the intercity passenger transport channel. He believed that in the duopoly market, railway enterprises and airlines should give full play to their own advantages, and pointed out that railway enterprises could significantly increase their market competitiveness by improving their train technology or adopting more favorable fares. Socrro and Viecens [4] based on the social benefits of airlines, including environmental factors, discussed the feasibility of high-speed rail aviation integration and the factors affecting its competitiveness, and analyzed its integration benefits by taking the Madrid-Malaga line as an example. With regard to freight flow research, Arunotayanun and Polak [5] take transportation time, cost, service quality, and service elasticity as the factors influencing shippers' behavior choice. By analyzing the output results of multinomial Logit model and mixed Logit model, it is found that the two results have different selection characteristics for different shippers to choose the mode of transportation. Talebian and Zou [3] applied the SP method, calibrated several logit model parameters, analyzed the competitive relationship among road, rail, and water transport in Rio Grande, Brazil, and pointed out that the most significant factor affecting multimodal transport is transport reliability, followed by transport cost, which provides the necessary reference for the development direction of local multimodal transport. Danielis et al. [6] take economy, rapidity, reliability, and safety as utility functions, apply the probit model to analyze the freight selection mode of Italian shippers, and point out that safety and reliability are more valued by shippers than transportation cost and timeliness.

Most of the existing studies are based on the utility function of customer or passenger choice to analyze the competitiveness of various transportation modes in the corridor. For the high-speed rail freight market, there is also a competition and cooperation relationship among the currently launched four transport products, namely, the same day, the next morning, the next day, and the third day. The freight flow sharing rate of the four products will affect the decision-making of the high-speed rail freight organization scheme. At present, the organization mode of highspeed rail freight restricts the railway carrying capacity. Therefore, in order to make full use of high-speed rail transport resources, we can guide demand through transport supply, and determine the cargo flow sharing rate of different high-speed rail freight products considering the relationship between supply and demand.

In the aspect of optimization of train transportation organization, the problem of making train operation plan is called LPP (line planning problem), in which line records the operation route of a train's OD, service frequency, marshalling, stop, and other information. When researchers 
and relevant staff evaluate whether the train operation scheme is reasonable, they mainly consider the following aspects:

(1) Whether it meets the demand of passenger and freight transportation.

(2) Whether convenient transportation service is provided.

(3) Whether the capacity (network, node, equipment, etc.) limitation is considered.

(4) Whether the relationship between cost and income is considered.

Therefore, in the study of the theory and method of train operation plan, many scholars focus on these aspects, consider the relationship between railway and customers, take one or more of the factors as the goal, consider a series of constraints such as demand satisfaction and network capacity, and construct the corresponding model and algorithm. Limited by the large scale of practical problems, various variables, complex application scenarios, and other factors, and at the same time to meet the requirements of the actual operation of the train operation scheme, the compilation method of train operation scheme has also changed from manual calculation to computer implementation.

Around the 1980s, researchers realized the importance of train operation plan, and initially used operational research theory to determine train operation plan. In this stage, the idea of iterative addition is widely used. For example, Sonntag [7] designed the optimization method of operation scheme with the goal of minimizing the total travel time of passengers. They designed an initial route set in advance, which requires the technical stations where the trains start or end to have relevant operation conditions. The iterative strategy of the algorithm is to delete some trains in the initial set, redistribute the passengers on the deleted trains to other trains with shorter travel time, and stop the iteration after meeting the requirements of the number of iterations or the shortest total travel time. We can see the rudiment of generating lining pool set from this idea, which lays an important foundation for future research.

Since the 1990s, with the increasing travel demand and more complex railway network scenarios, more and more researchers have established mathematical optimization models to solve more complex optimization problems of train operation schemes. In this stage, a large number of mathematical optimization models based on different optimization strategies have emerged, which can be divided into two categories: one is the optimization model of train operation scheme considering the lowest train operation cost from the perspective of road network company [8], and the other is the optimization model of train operation scheme considering the shortest passenger travel time from the perspective of passenger travel angle [9]. Most of the subsequent studies follow the ideas of these two papers, which can be summarized as follows.

2.1. Optimization Model of Train Operation Plan considering Operation Cost. $G(S, A, F)$ is the directed graph of railway network, $S$ is the station set, $A$ is the train running arc set, and $F$ is the train set. On the premise that the service network meets the needs of passenger travel, the train operation method considering the operation cost is to minimize the cost of providing transportation services.

$C_{A}$ indicates the carrying capacity of the section

$q$ Passenger flow

$L$ Train candidate set

$f$ Service frequency

$r$ Number of train formation

$k$ Operating costs

$x_{L} 0-1$ variable indicates whether a scheme in the alternative set is selected

Obj:

$$
\min Z=\sum_{L} x_{L} \cdot k_{L}
$$

s.t.

$$
\begin{gathered}
f \cdot x_{L} \leq C_{A}, \\
f \cdot r \cdot x_{L} \leq q, \\
x_{L} \in\{0,1\} .
\end{gathered}
$$

Among them, formula (1) indicates the lowest railway operation cost. Formula (2) indicates the section capacity constraint. Formula (3) indicates that the transportation demand meets the constraint. Formula (4) indicates the value range of decision variables.

Based on this kind of model, Assad [10] takes the minimum total transportation cost as the goal, and takes the operation frequency, train operation path, and interval as variables to construct the optimization model of train operation scheme. On this basis, Classens [11], Racunia and Wynter [12], Ertem and Keskin Özcan [13], and other scholars have considered more comprehensive factors, such as line network capacity, service object, train operation condition, train bottom operation, etc., constructed a train operation scheme formulation model, and designed intelligent optimization algorithm to solve it. In algorithm design, researchers use branch and bound, cut plane method, and intelligent optimization algorithm to solve the problem $[14,15]$. 
2.2. Optimization Model of Train Operation Scheme considering the Shortest Passenger Travel Time. Obj:

$$
\min Z=\sum_{L} x_{L} \cdot t_{L}
$$

s.t.

$$
\begin{gathered}
f \cdot x_{L} \leq C, \\
f \cdot r \cdot x_{L} \leq q, \\
\cdots, \\
x_{L} \in\{0,1\} .
\end{gathered}
$$

Among them, $t_{L}$ is the time taken by passengers in each stage, and formula (5) is the shortest travel time. Formula (6) represents the section capacity constraint. Formula (7) shows that the passenger flow demand meets the constraint. Formula (8) can represent the constraints of other factors, because there are many dimensions to analyze the total travel time of passengers (travel time, transfer times, safety and economy, etc.). Formula (9) represents the value range of decision variables. It can be seen that the factors considered in the model considering passenger travel time are similar to those in the model considering railway operation cost, but the output train operation schemes of the two models are quite different due to different objective functions and special constraints.

Based on this kind of model, Bussieck et al. [16] constructed a mixed-integer programming model with the goal of maximizing the direct passenger traffic, considering the constraints of equations (6) and (7), and applied the operational research method to solve the model after dealing with the train capacity constraints with many variables. Schöbel [17] studied the train operation plan making method with the objective of minimizing the number of passenger transfers, and considered the operation cost as the constraint condition. Similarly, based on the idea of train alternative set, a passenger transfer network is constructed. Taking whether a train is selected and the relationship between interval and route as decision variables, the spatiotemporal path of passengers in the constructed transfer network is depicted. Because of the huge scale of solution, the author adopts heuristic algorithm and precise algorithm to solve the problem.

In order to increase the number of passenger trains on Double-Track railway, Jiang et al. [18]studied the comprehensive optimization of train operation scheme and stop scheme based on the busy trunk line of high-speed railway. To meet the continuous growth of passenger demand, it should add some trains on the basis of the existing timetable usually. In order to achieve this goal, they discussed the decision-making methods such as increasing the stop time of some trains at some stations or adjusting the stop strategy.

In addition to the comprehensive optimization of train operation scheme and timetable, some scholars analyzed the robustness of train operation scheme, and analyzed the influence of interval, stop, delay, and other factors on transport services, aiming at reducing the total travel time of passengers. For busy trunk lines with capacity close to saturation, Burggraeve and Vansteenwegen [19] constructed a train diagram optimization model considering the occupation of railway facilities and equipment (such as block section) by trains, and designed a solution algorithm. Taking Brussels road network as an example, the effectiveness of the model and algorithm was verified.

Cacchiani et al. [20] compared and analyzed the solution quality of the exact solution strategy and the heuristic algorithm solution strategy in the train service planning problem. In this paper, the robustness of train diagram and the adjustment strategy of train diagram delay are systematically described, an integer programming model of train diagram compilation based on the shortage of railway transportation network capacity is constructed, and a heuristic algorithm is designed to solve the model, so as to realize the rapid solution of train diagram compilation.

In order to achieve the optimal operation strategy of the operation network, Qi et al. [21] constructed a multi-objective mixed-integer programming optimization model based on the train operation interval, stop plan, and passenger flow distribution, taking the shortest deadhead mileage and the least number of stops as the optimization objectives, and applied gams to realize two case studies, which proved the feasibility of the proposed method.

Combined with the actual railway network conditions, Claessens et al. [11] constructed a nonlinear mixed certificate planning model with minimum operation cost. The model considers the factors such as train operation path, train speed level, and service frequency. In order to achieve a good solution, the nonlinear constraints in the model are transformed into linear constraints, and the classical exact solution strategy is adopted.

Wang [22] deeply analyzed the passenger demand and transportation organization mode of extra-long line. Implement the method of transit assignment for high-speed railway network considering passengers' whole process of travel. Put forward direct and transfer transportation mode for passenger flow with extra-long distance.

Wang et al. [23] built a train service network according to the characteristics of the Dutch railway network. There are three types of trains in the network, namely AR, IR. and IC trains. These three trains meet different travel requirements of passengers, and the service scope of each train is significantly different. Similarly, Wong et al. [24] has studied the allocation of urban roads based on the characteristics of route selection. Pfetsch and Borndrfer [25] studied the problem of passenger travel route selection based on the multi-commodity flow model. The Bertsimas and Sim [26] study evaluated the robustness of the results of the train operation plan. Torres et al. [27] studied the optimization problem of the train operation plan, and built a mixedinteger programming model with the goal of minimizing the total train operation cost.

To sum up, the research on the theory and method of high-speed rail freight organization scheme is still blank in China. In terms of optimization modeling, the model based on train candidate set has some advantages in solving quality 
and efficiency. The preparation of high-speed rail freight organization plan should be oriented to the demand of fast transport freight flow, and adapt to the multidimensional requirements of express market for transport service flow, timeliness, quality, and so on.

Combined with the future planning of China's highspeed railway operation organization mode, we will focus on the research of market demand and the impact of demand on transport supply. Firstly, the calculation method of highspeed rail freight flow sharing rate considering supply-demand matching relationship is designed. Based on this, a two-stage model of high-speed rail freight organization scheme optimization is constructed. In the first stage, the model of train alternative set is established, which takes the minimum total cost of train operation as the objective, and considers the line capacity, section flow, and OD demand, so as to reduce the scale of train alternative set and improve the efficiency of solution. In the second stage, an accurate product demand allocation model with the objective of optimal economic benefit is established, and a high-speed rail freight organization scheme considering freight flow demand and transportation network capacity is generated.

\section{Research on Freight Flow Sharing Rate of High-Speed Railway}

3.1. Problem Description. At present, high-speed rail freight has mainly launched four kinds of transport products, namely, Same-day delivery ( $\mathrm{d}$ for short in the following text), Next Morning Delivery (c for short in the following text), Next Day Service ( $\mathrm{r}$ for short in the following text), and the third-day delivery ( $\mathrm{s}$ for short in the following text). By calculating the sharing rate of four kinds of transportation products, the transportation demand of different transportation products can be determined, and then the transportation supply strategy can be optimized according to the transportation demand.

The calculation of freight flow sharing rate of the freight product can be divided into two stages. In the first stage, the freight flow sharing rate of the freight product is calculated when the supply capacity is sufficient, that is, when the supply can fully meet the demand, the freight flow sharing rate of the freight product is calculated based on the customer's choice intention. The second stage is to calculate the transfer of freight flow between products when some transportation products cannot meet the demand effectively due to the limitation of transportation organization mode and transportation capacity. The freight flow sharing rate of the freight product calculated in the first stage can be used as the input parameter for comprehensive optimization of high-speed rail freight train diagram compilation, and the freight flow transfer sharing rate calculated in the second stage can be used as the basis for matching high-speed rail freight trains with the freight product.

At present, several products launched by high-speed rail freight are more cooperative. At this stage, the organization mode of high-speed rail freight limits the capacity of highspeed rail freight. Therefore, in order to make full use of high-speed rail transportation resources, we can guide demand through transportation supply, and determine the freight flow sharing rate of different freight products by considering the relationship between supply and demand.

This section will analyze the relationship between customers' choice behavior and the supply of the freight product. A two-stage calculation method of freight flow sharing rate of the freight product based on supply and demand matching has been designed. In the first stage, considering the customers' willingness to choose the freight product, the utility function of the freight product is constructed, and the calculation model of freight flow sharing rate of the freight product considering the types of freight flow is established, in order to describe the influence of the differences of customers' choice behavior and freight flow structure on the freight flow sharing rate. In the second stage, considering the supply characteristics of the freight product, the calculation method of freight flow transfer probability of the freight product related to customer selection utility value is designed, and the calculation result of freight flow sharing rate in the first stage is modified.

3.2. Construction of High-Speed Rail Freight Flow Sharing Rate Model Based on Customer Choice Intention. The choice of the freight product varies greatly with different attributes of freight flow, so we first classify the freight flow of the freight product, and then analyze the technical and economic characteristics of different products, and then build a Logit model considering the types of freight flow and the utility value of the freight product.

3.2.1. Classification of High-Speed Rail Freight Flow. It is suitable for the use of high-speed rail freight goods, mainly including letters, clothes, serum, vaccines, flowers, electronic accessories and books, daily necessities, and so on. It is found that the higher the value of goods, the less sensitive they are to time. The key factors that affect customers' choice of the freight product are the value of goods and time sensitivity. The different attributes of goods will also affect the utility value of various transport products. Therefore, the highspeed rail freight flow is classified according to these two aspects. Based on the investigation of the price, time, stability, and other factors of freight products, the system clustering method is used for analysis, and the results are shown in Table 1.

In Table 1, each number represents the code of this kind of goods. The classified freight flows can be divided into three categories: 1, 2, and 4 are high-end freight flows, which are characterized by their high value and high requirements for transportation timeliness, such as certificates, medical surgery, and urgently needed drugs; 3,5 , and 7 are midrange freight flows, which are characterized by their high value or high timeliness requirements, such as letters, vaccines, and other goods; and 6, 8, and 9 are low-end freight flows, which are characterized by their high value and low timeliness requirements, such as clothing, books, and other goods. For example, the freight flow with code number 1 has high property value and high timeliness requirements, that is, serum, certificates, organs, and other goods. When 
TABle 1: Classification of high-speed rail freight flow.

\begin{tabular}{lccc}
\hline Value attribute & \multicolumn{3}{c}{ Time attribute } \\
& Sensitive & Relatively sensitive & Insensitive \\
\hline High & 1 & 2 & 3 \\
Moderate & 4 & 5 & 6 \\
Low & 7 & 8 & 9 \\
\hline
\end{tabular}

customers choose to transport products, the preferred product is the same day delivery. However, if the service time of the same day delivery product has exceeded, the freight flow will be transferred. Other examples are clothing, books, and other goods, which can be summed up as code 8 or 9. If there is no special case, customers will choose transportation products with lower price, that is, second day or the third day delivery. However, if the books need to be used urgently, they can be considered as freight flow code 4. Therefore, through statistical analysis of the main categories of goods and the transport products selected by different categories of goods, we get Table 1 . In other words, Table 1 is only a statistical analysis of the existing status, not an accurate calculation result. Because the transportation demand is relatively stable over a period of time, even if the demand for transportation products by different goods categories fluctuates, the transportation products can still be designed through the freight flow allocation method we studied, which can still improve the service level of high-speed rail freight.

3.2.2. Utility Function. The utility function value of transportation products can approximately represent the perceived utility of customers and determine their probability of being selected. Generally, the utility function value of transportation products is related to its economy, rapidity, convenience, safety, punctuality, and other factors. For all kinds of freight products, there is little difference in safety and punctuality. Therefore, we only consider the impact of economy, rapidity, and convenience on customers' choice behavior. Because different freight flows have different demands for the freight product, the difference of the freight product should be considered when calculating the utility function value, as shown in equation (10).

$$
V_{\text {in }}=\left(\theta_{1 n} J_{\text {in }}+\theta_{2 n} K_{\text {in }}-\theta_{3 n} C_{\text {in }}\right) .
$$

Among them, $V_{\text {in }}$ represents the utility value of class $i$, the freight product to class $n$ freight flow. $J_{\mathrm{in}}, K_{\mathrm{in}}$, and $C_{\mathrm{in}}$ represent the utility value of class $i$, the freight product to class $n$ freight flow economy, rapidity, and convenience. $\theta_{1 n}$, $\theta_{2 n}$, and $\theta_{3 n}$ represent the unknown parameters of economy, rapidity, and convenience to classify a freight flow, respectively.

The economy of various freight products can be determined according to the charging standards of various products, as shown in equation (11).

$$
J_{\text {in }}=S_{i}+X_{i} \cdot \bar{g}_{i} .
$$

Among them, $S_{i}$ is the beginning of class $i$, the freight product, that is to say, those below a certain weight will be charged a uniform fee. $X_{i}$ is the excess weight of class $i$, the freight product, that is, if the goods exceed $S_{i}$, the excess is charged in multiples of a certain weight. And, $g_{i}$ is the average excess weight of different types of goods.

The rapidity of all kinds of freight products can be determined according to the total service time of all kinds of products, including the transportation time and waiting time of goods, as shown in equation (12).

$$
K_{\text {in }}=T+\bar{W}_{\text {in }} .
$$

$T$ represents the transportation time of goods, $W_{\text {in }}$ represents the average time of class $n$ goods flow waiting for class $i$ goods to depart and deliver.

Limited by the current transport organization mode, in order to avoid interference to passenger transport, only a fixed number of trains are selected to carry out high-speed rail freight business. The convenience of various freight products can be expressed by the number of trains that can bear such products in one day:

$$
C_{\text {in }}=N_{i} \text {. }
$$

Among them, $N_{i}$ is the number of trains that can undertake the task of class $i$, the freight product in one day.

3.2.3. Calculation Model of Freight Flow Sharing Rate of High-Speed Rail Freight Products. In the traditional Logit model, the probability of a certain transportation product being selected is determined by the absolute difference of utility among the products. When the generalised cost is high, some unreasonable results will be produced. Therefore, this paper uses relative utility to calculate the selection probability of all kinds of freight products, and uses $P_{\text {in }}$ to express the selection probability of class $i$, the freight product by class $n$ freight flow:

$$
P_{\text {in }}=\frac{\exp \left(V_{\text {in }} / \bar{V}\right)}{\sum_{j} \exp \left(V_{j n} / \bar{V}\right)}
$$

where $\bar{V}$ is the average value of utility function.

On this basis, further considering the proportion of various types of freight flow in the corridor, the freight flow sharing rates of different freight products can be obtained, as shown in the following formula:

$$
\begin{aligned}
P_{i} & =n_{1} P_{i 1}+n_{2} P_{i 2}+n_{3} P_{i 3}, \\
n_{1}+n_{2}+n_{3} & =1 .
\end{aligned}
$$

Among them, $P_{i}$ represents the freight flow share rate of class $i$, the freight product, and $n_{1}, n_{2}$, and $n_{3}$ represent the 
proportion of three levels of high-speed rail freight flow in the corridor, respectively.

3.3. Construction of Freight Flow Sharing Rate Model of HighSpeed Rail Freight Products considering Supply Capacity. Limited by the characteristics of high-speed rail freight organization and transportation capacity, when the customer's choice intention cannot be satisfied, the customer will choose other similar freight products. Therefore, the transfer of customer's intention is related to the utility function value of each high-speed rail freight product. In Section 3.2, we study the freight flow sharing rate of the freight product based on customers' choice intention, without considering the influence of the supply characteristics of the freight product on the freight flow sharing rate of products. This section will analyze the impact of the supply capacity of the freight product on customers' choice behavior. Firstly, the relationship between each type of freight flow transfer and the utility value of the freight product is analyzed, and then the total probability of freight flow transfer is calculated, so as to obtain the freight flow sharing rate of various types of freight products. This paper assumes that there is no backlog of goods.

3.3.1. Transfer Conditions of High-Speed Rail Freight Products. Let $\mathrm{N}$ represent all trains that can undertake the high-speed rail freight task in the corridor; Ni represent the number of trains that undertake $\mathrm{i}$, the freight product; $\mathrm{Q}$ represent the total demand of high-speed rail freight market; $\mathrm{C}$ represent the total loading capacity of high-speed rail freight trains; $\Delta_{i}$ represent $\mathrm{i}$, the freight product, if there is a difference between the demand of class $i$, the freight product, and the actual carrying capacity. The transfer conditions of the high-speed rail freight flow from freight products class $i$ to class $\mathrm{j}$ can be expressed as follows:

$$
\left\{\begin{array}{l}
\Delta_{i \longrightarrow j}=\frac{Q \cdot P \cdot N_{i}}{C \cdot N_{i}}-1, \\
\Delta_{i \longrightarrow j}>0, \\
\Delta_{j}<0 .
\end{array}\right.
$$

Equation (17) indicates that only when the probability of a certain product being selected multiplied by the demand is greater than the value of the opening ratio multiplied by the total carrying capacity of the high-speed rail, the flow of goods will shift.

3.3.2. Calculate the Transfer Probability of Freight Flow between Different Products. When a certain kind of transportation product cannot meet the customer's choice intention, other transportation products should be considered. Take the example of the same day delivered; if the customers cannot choose the products delivered on the same day due to the influence of transportation capacity, transportation organization, mode, and other factors, this part of the freight flow will be transferred, as shown in equation (18).

$$
P_{i \longrightarrow j}^{n} \propto \frac{1}{V_{i}^{n}-V_{j}^{n}} .
$$

Among them, $P_{i \longrightarrow j}^{n}$ represents the probability of freight flow transferring from class $i$, the freight product, to class $i$ products; $V_{i}^{n}$ and $V_{j}^{n}$ represent the utility function values of the two types of products, respectively.

It can be seen from equation (18) that the probability of freight flow transfer is related to the utility value of all kinds of freight products, and customers will give priority to freight products with similar utility value to the products they choose. Therefore, in order to determine the probability of freight flow transfer, it is necessary to establish the expression of probability and utility value. According to the positive and negative of $V_{d}^{n}-V_{j}^{n}$, it can be divided into the following three cases:

(1) $V_{d}^{n}-V_{j}^{n}>0$

It means that the utility value of other freight products is lower than that of the current day arrival products, and the freight flow transfer probability can be expressed as follows:

$$
\begin{aligned}
& P_{d \longrightarrow c}^{n}+P_{d \longrightarrow r}^{n}+ P_{d \longrightarrow s}^{n}=1-P_{d}^{\prime}, \\
& \frac{P_{d \longrightarrow c}^{n}}{P_{d \longrightarrow r}^{n}}=\frac{V_{d}^{n}-V_{c}^{n}}{V_{d}^{n}-V_{r}^{n}} \\
& \frac{P_{d \longrightarrow r}^{n}}{P_{d \longrightarrow s}^{n}}=\frac{V_{d}^{n}-V_{r}^{n}}{V_{d}^{n}-V_{s}^{n}}
\end{aligned}
$$

(2) $V_{d}^{n}-V_{j}^{n}<0$

It indicates that the utility value of other freight products is higher than that of the current day arrival products, and the probability of freight flow transfer can be expressed as follows:

$$
\begin{aligned}
& P_{d \longrightarrow c}^{n}+P_{d \longrightarrow r}^{n}+ P_{d \longrightarrow s}^{n}=1-P_{d}^{\prime}, \\
& \frac{P_{d \longrightarrow c}^{n}}{P_{d \longrightarrow r}^{n}}=\frac{V_{d}^{n}-V_{r}^{n}}{V_{d}^{n}-V_{c}^{n},} \\
& \frac{P_{d \longrightarrow r}^{n}}{P_{d \longrightarrow s}^{n}}=\frac{V_{d}^{n}-V_{s}^{n}}{V_{d}^{n}-V_{r}^{n} .}
\end{aligned}
$$

(3) $V_{d}^{n}-V_{j}^{n}>0$ or $V_{d}^{n}-V_{j}^{n}<0$

It shows that the utility value of some transportation products is higher than that of the current day, and some are lower than that of the current day. At this time, only the case of $V_{d}^{n}-V_{j}^{n}>0$ is considered, and the freight flow is transferred in direct proportion to the utility value, and the transfer probability of $V_{d}^{n}-V_{j}^{n}<0$ is 0 .

Let $\alpha_{i \rightarrow j}$ represent the total probability of goods flow transferring from product $i$ to product $j$, which can be expressed as: 


$$
f_{i \longrightarrow j}=\alpha_{i \longrightarrow j} \cdot \Delta_{i}
$$

3.3.3. Modified Calculation of Freight Flow Share Rate of Various Products. According to the criterion formula (17), there are two cases of freight flow transfer:

(1) $\left|\Delta_{j}\right|>f_{i \rightarrow j}$

$$
\begin{aligned}
& P_{i}^{\prime}=P_{i}-f_{i \longrightarrow j}, \\
& P_{j}^{\prime}=P_{j}+\sum_{1}^{i, i \neq j} f_{i \longrightarrow j} .
\end{aligned}
$$

(2) $\left|\Delta_{j}\right|<f_{i \longrightarrow j}$

$$
\begin{aligned}
P_{i}^{\prime} & =P_{i}-f_{i \longrightarrow j}, \\
P_{j}^{\prime} & =P_{j}+\Delta_{j}, \\
P_{k}^{\prime} & =P_{k}+\min \left\{\Delta_{k}, f_{i \longrightarrow k}\right\}, \\
P_{l}^{\prime} & =P_{l}+\min \left\{\Delta_{l}, f_{i \longrightarrow l}\right\} .
\end{aligned}
$$

\section{Research on Freight Flow Sharing Rate of High-Speed Railway}

4.1. Problem Description. After defining the freight demand, we need to firstly determine the service range of high-speed rail freight. High-speed rail express service path optimization problem is a kind of large-scale, variable combination of space-time resource optimization allocation problem; for this kind of combination optimization problem, it is difficult to obtain the optimal solution through direct calculation. Therefore, before determining the organization scheme of high-speed rail express, we should first eliminate some cases that obviously do not conform to the principles of the organization scheme, in order to reduce the computational complexity and the scale of the problem in the distribution stage. They mainly include: some schemes do not fully consider the limitation of network capacity, some busy sections are short of transport capacity resources, and they do not have the ability to operate freight trains; they do not fully consider the relationship between trains, resulting in repeated operation in some sections, resulting in waste of resources; they do not consider the coupling relationship between freight flow and products, resulting in excessive waste of section capacity. In order to solve the above problems, we should first construct the optimization model of train service path, and construct a service network to meet the conditions of freight train operation, economic benefits, and freight flow demand, that is, to screen out a reasonable and feasible train service path, and then subdivide it into service products and modes in the next stage based on the alternative train service path, so as to realize the accurate distribution of freight flow.

4.2. Model Hypothesis. This paper assumes that the train runs on the double track railway network. Combined with the reality of railway organization plan, the important constraint of high-speed rail express business is the lack of matching facilities and equipment at stations. With the expansion of business, the railway will also build or transform the facilities at stations to facilitate the organization of cargo loading and unloading, handling, and storage. Therefore, it is assumed that each parking station has the conditions and sufficient capacity to organize high-speed rail express business. At present, the parameters of freight multiple units are the same as those of passenger multiple units. Assuming that the grade of high-speed rail express train is the same as that of passenger train, the operation of freight multiple units is not considered.

The inputs of the model are: fixed cost of train operation, variable cost related to distance, stop cost related to train start and stop, section capacity, freight flow demand, and train carrying capacity. The output of the model is the optimized train alternative service path and combined stop mode, which can meet the market demand.

\subsection{Model Formulation}

\subsubsection{Notation}

Sets

$F$ : Set of the trains.

$\Omega$ : Set of the OD demand.

$\Omega_{j k}$ : Set of the sections that the train needs to pass through.

$\phi$ : Set of the stations.

$\varepsilon$ : Set of the sections.

Parameters

$Q_{\omega}$ : Total flow of goods between $\omega$.

$C_{\omega}$ : Unit weight freight of $\omega$.

$c_{i}^{F}$ : Fixed cost of train operation.

$c_{i}^{V}$ : The unit distance variable cost of train operation.

$c_{i}^{t}$ : One stop cost of train $i$ operation.

$d_{i}$ : Running distance of train $i$.

$C_{i}$ : Loading capacity of train.

$C_{j k}$ : Section capacity.

$V_{i}$ : The load of the train $i$.

$M$ : Infinite positive number.

Decision Variables

$x_{i}^{s}$ : Equals 1 if the train $i$ stops at station $s, 0$ otherwise.

$y_{i}^{\omega}$ : Equals 1 if the train $i$ serves $\omega, 0$ otherwise.

$z_{j k}^{i}$ : Equals 1 if the train $i$ passes though the section $(i, j), 0$ otherwise.

$f_{\dot{j}}:$ The operating frequency of train $i$.

$k_{s}^{\delta_{\omega} / D_{\omega}}$ : Equals 1 if the station $s$ is the origin or destination of $\omega, 0$ otherwise.

4.3.2. Objective Function. While establishing the service route optimization model, it is necessary to eliminate the train operation types that obviously do not conform to the 
principle of organization scheme compilation, and the direct consequence of violating the principle of operation scheme compilation is that the train operation cost is too high. Therefore, it is necessary to focus on the train operation cost at this stage. At the same time, it is necessary to ensure that the train candidate set can meet the demand of freight transportation. Therefore, this paper takes the lowest train operation cost as the goal, and constructs the generation model of high-speed rail freight train selection set.

$$
\operatorname{Min} Z_{1}=\sum_{i \in F} f_{i} \times\left(c_{i}^{F}+\sum_{(j, k) \in \varepsilon} c_{i}^{V} \cdot z_{j k} \cdot d_{j k}+\sum_{s \in \phi} x_{i}^{s} \cdot c_{i}^{t}\right) .
$$

Equation (24) shows that the operation cost of highspeed rail freight train is the lowest, which can be divided into three parts: fixed cost of train operation, variable cost related to distance, and variable cost related to train stop. In order to minimize the total cost of train operation and effectively meet the freight demand, it is necessary to ensure the optimality of freight train operation interval selection and stop strategy.

\subsubsection{Constraints}

(i) Section Capacity Constraint. Section capacity is related to fixed equipment. In addition, the carrying capacity of highspeed railway freight trains is also affected by the mode of transportation organization. At present, high-speed railway is still dominated by passenger transportation, and highspeed railway freight trains can only operate on the basis of not affecting passenger transportation. Therefore, the amount of space-time resources left for high-speed railway freight trains is based on the busy degree of high-speed railway passenger transportation.

Furthermore, the busy degree of each section is different, which leads to different resources for high-speed rail freight trains. In order to make full use of the limited capacity resources of high-speed railway, it is necessary to fully consider the difference of section capacity and reasonably determine the type of train operation when compiling the operation scheme of high-speed railway freight train. The schematic diagram of section capacity constraint is shown in Figure 2.

Among them, train 1 , train 2, and train $i$ indicate the trains passing through the section a-e, the broken line indicates the maximum bearing capacity of the section. As can be seen from Figure 3, the capacity constraint of a certain section can be expressed as: if the total number of passing trains cannot be greater than the capacity of the section, it can be expressed as

$$
\sum_{i \in F} f_{i} \cdot z_{j k}^{i} \leq C_{j k}, \quad \forall(j, k) \in \varepsilon .
$$

(ii) Train Running Distance Constraint. As mentioned above, if the starting and ending points of trains are too close, the fixed cost of train operation will increase, and in order to

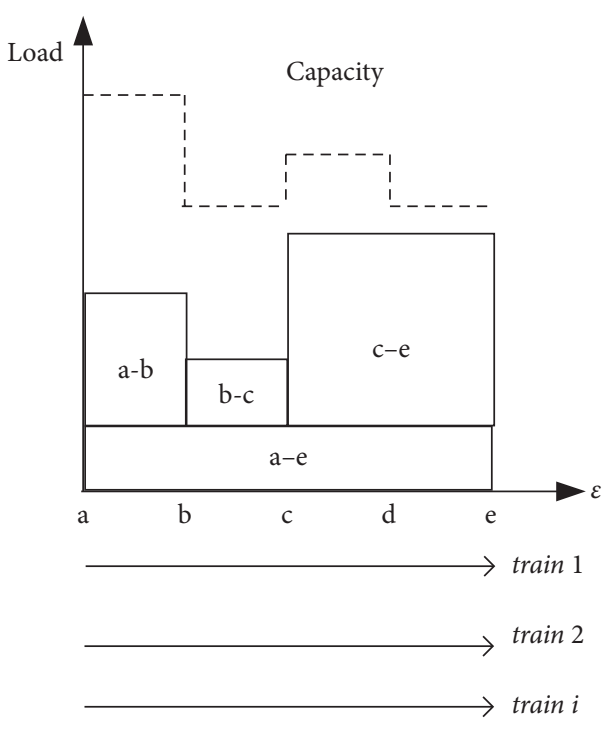

FIGURE 2: The constraint of the section capacity ability.

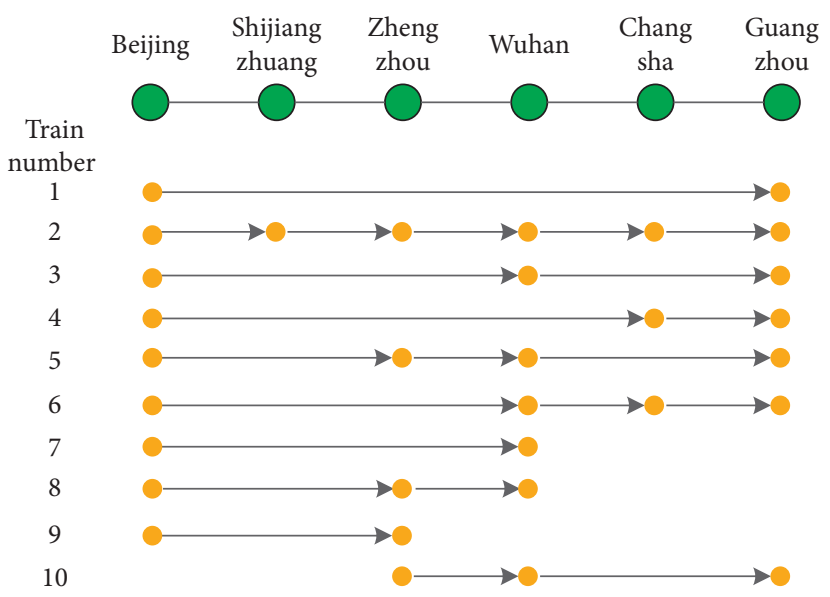

Figure 3: The backup plan for high-speed rail express trains in the down direction.

effectively meet the total freight demand, it is bound to increase the number of high-speed rail freight trains operating in other sections. Therefore, the train running distance constraint can be expressed as

$$
\begin{aligned}
v_{i} \leq f_{i}, \quad \forall i \in F, \\
f_{i} \leq v_{i} \cdot \mathbf{M}, \quad \forall i \in F, \\
\sum_{(j, k) \in \varepsilon} v_{i} \cdot z_{j k} \cdot d_{j k} \geq D_{i}, \quad \forall(j, k) \in \varepsilon, \forall i \in F .
\end{aligned}
$$

Among them, $v_{i}$ is the $0-1$ auxiliary variable, and expressions (26) and (27) represent the mapping constraints of auxiliary variables, changing the train operation frequency to whether to operate, and $\mathbf{D}$ represents the correlation matrix of the minimum running distance of the train. 
(iii) Demand Satisfaction Constraints. The volume of cross section flow of goods depends on the loading capacity of the train, that is, the total amount of goods carried by the train in the cross section of each train running path cannot exceed the limit of the loading capacity of the train. Different sections may have different requirements for train loading capacity. In order to meet the demand of freight transportation, it is necessary to ensure that the product of train service frequency and loading capacity is greater than the total demand, that is to say, in each section of the train running path, the total freight volume of each high-speed rail freight train cannot exceed its rated capacity, which requires us to reasonably design the loading plan of freight trains to ensure that the demand of each section can be met.

$$
\frac{\sum_{\omega} q \cdot z_{j k}}{V_{i}} \leq f_{i}, \quad \forall \omega \in \Omega .
$$

(iv) Train Assignment Constraints. Equation (29) gives the constraint that the total loading capacity of the train can meet the transportation demand, but the freight transportation demands specific OD should also be assigned to specific high-speed rail freight trains.

$$
\sum_{i \in F} f_{i} \times y_{\omega}^{i} \times M \geq Q_{\omega}, \quad \forall \omega \in \Omega .
$$

\section{Algorithm Design}

The column generation algorithm is developed from the simplex algorithm for solving linear programming. For the solution of the train service path problem, each "train" represents the train with different starting and ending points and stop schemes. In the iterative process, if the train generation algorithm cannot effectively meet the requirements or train carrying capacity and other constraints, it will "add trains." Each additional train means that trains with different types of feasible solutions are added. As the iterative process continues to "add trains," it will continue to optimize the feasible solution of the original problem until the subproblem cannot "add trains," which means that there is no better type of trains, and then the solution is the optimal solution.

Similar to the simplex method, when using the column generation algorithm to solve the train candidate set model, we should first construct the test number subproblem of the original problem. According to the principle of the train generation algorithm, the variables appearing in the "train" position are all parameters related to the train and the interval. Therefore, each additional "train" actually represents an additional train. By continuously adding trains in the interval, we can judge whether it can effectively meet the demand of goods supply and ensure the train operation cost. Therefore, in the alternative set model stage of the original problem, we consider the limitation of the network, that is, the problem of which section to run and which station to stop at has the lowest cost. In the inspection number subproblem, we need to consider the limitation of each train, so we include the factors of train stop, train carrying capacity, train route, and so on. The subproblem model of test number is constructed as follows.

\subsection{Objective Function.}

$$
\begin{aligned}
\operatorname{Min}_{2}= & \left(\sum_{i \in F} c_{i}^{F}+\sum_{(j, k) \in \mathcal{\varepsilon}} c_{i}^{V} \cdot z_{j k} \cdot d_{j k}^{i}+\sum_{s \in \phi} x_{s} \cdot c_{i}^{t}\right)- \\
& \sum_{(j, k) \in \mathcal{\varepsilon}} \pi_{j k} \cdot z_{j k}-\sum_{j k} \mu_{j k} \cdot z_{j k} \cdot d_{j k} \\
& -\sum_{(j, k) \in \mathcal{\varepsilon}} \frac{\rho_{j k} \cdot \sum_{\omega} q \cdot z_{j k}}{V_{i}}-\sum_{\omega \in \Omega} \eta_{\omega} \cdot y_{\omega} \cdot M .
\end{aligned}
$$

Among them, $\pi_{j k}, \mu_{j k}, \rho_{j k}$, and $\eta_{\omega}$ represent the simplex multipliers of equations (25), (28)-(30), respectively, which represent the value of different resources.

\subsection{Constraints}

5.2.1. Service and Stop Relationship Constraints. It is necessary to ensure that when train $i$ serves OD pair $\omega$, it should stop at the starting point and terminal of $\omega$. Therefore, the relationship between service and stop can be expressed as follows:

$$
\begin{aligned}
y_{i}^{\omega} \leq x_{i}^{O / D_{\omega}}, \quad \forall \omega \in \Omega, \forall i \in F, \\
y_{\omega}+\left(2-x_{O_{\omega}}-x_{D_{\omega}}\right) \geq 1, \quad \forall \omega \in \Omega .
\end{aligned}
$$

Equations (32) and (33) denote the relationship between train service and origin destination, that is, if a train serves an OD pair, it must stop at its origin and destination.

5.2.2. Train Stop Times Constraint. As mentioned above, if the train stops too many times, it will reduce the travel speed of goods, increase the train operation cost, and occupy more railway network resources. Therefore, the number of train stops should be limited.

$$
\sum_{s \in \phi} x_{i}^{s} \leq \alpha, \quad \forall i \in F
$$

Among them, $\alpha$ is a constant, which means that the number of train stops cannot exceed a known constant.

5.2.3. Train Stop and Starting and Destination Point Constraint. Firstly, we need to ensure that there is only one starting point and end point. Secondly, the train serving the OD must stop at the starting point and terminal point of this OD pair, the expressions are as follows: 


$$
\begin{gathered}
\sum_{s \in \delta} k_{s}^{O} \times s \leq \sum_{s \in \delta} k_{s}^{d} \times s, \\
2-x_{i}^{O_{\omega}}-x_{i}^{D_{\omega}} \leq 0, \quad \forall \omega \in \Omega, \forall i \in F, \\
\left(1-k_{s}^{O / D}\right)+1 \geq x_{s}, \quad \forall s \in \delta .
\end{gathered}
$$

Equation (35) indicates that the train has only one starting point and one ending point. Equations (36) and (37) indicate the relationship between the train stop and OD, that is, the train needs to stop at the original and destination of the freight transportation demand.

5.2.4. Train Running Path Constraint. In order to constrain the train's running path, first of all, it is necessary to ensure that the stop of the train will not exceed the range of $O D$, and secondly, the train passing section cannot be outside OD yet, the expressions are as follows:

$$
\begin{aligned}
& \sum_{n=1}^{s-1} x_{i}^{s-n} \leq\left(1-k_{s}^{O_{\omega}}\right) \cdot M, \quad \forall s \in \phi, \forall \omega \in \Omega, \forall i \in F, \quad \\
& \sum_{n=1}^{\phi-s} x_{i}^{s+n} \leq\left(1-k_{s}^{D_{\omega}}\right) \cdot M, \quad \forall s \in \phi, \forall \omega \in \Omega, \forall i \in F, \quad \text { (39) } \\
& z_{j, k}-\sum_{s \in \delta: s \leq j} k_{s}^{O}-\sum_{s \in \delta: s \leq j} k_{s}^{d} \leq-1, \quad \forall(j, k) \in \varepsilon .
\end{aligned}
$$

Among them, Constraints (38) and (39) indicate that no stops are allowed outside the origin and destination. Constraint (40) indicates the relationship between the train passing section and the origin and destination point, that is, the train cannot go through the section beyond the origin destination point.

\subsubsection{Decision Variables Constraint.}

$$
\begin{array}{ll}
0 \leq x_{i}^{s} \leq 1, & x_{i}^{s} \in N, \\
0 \leq y_{i}^{\omega} \leq 1, & y_{i}^{\omega} \in N, \\
0 \leq z_{j r}^{i} \leq 1, & z_{j r}^{i} \in N, \\
0 \leq k_{s}^{O_{\omega}} \leq 1, & k_{s}^{O_{\omega}} \in N .
\end{array}
$$

\section{Establishment of Distribution Model considering Different Product Requirements}

6.1. Problem Description. At present, the organization modes of high-speed rail express include confirm train, passenger train piggyback transportation, reserved train carrier, and high-speed rail express. Among them, the confirmed train refers to the first train that does not carry people every morning. This train is only for confirming the safety of the line, so it can be used to carry express delivery. Passenger train piggyback transportation refers to the mode of using the remaining space of passenger trains to transport express delivery. In the reserved train carriage mode, the passenger train carriage is reserved as a whole, without selling tickets, and is specially used for express transportation. At the same time, under the above four organization modes, high-speed rail express includes four transportation products. Therefore, the problem of freight flow allocation considering product demand can be refined into the matching problem of different organization modes and different transportation products.

By considering the relationship between train stop, interval, and demand, the service route of train is determined, and a framework operation scheme which can meet the market demand, including train operation type and stop combination is generated. However, it only expresses which kinds of trains can be operated, and does not select specific trains or allocate freight flow to trains. This section is based on the current four kinds of transportation products and four kinds of organization mode to segment the market demand, so as to realize the accurate distribution of freight flow.

In the flow allocation stage, it is assumed that each OD pair can meet the operation requirements of different organization modes, and has the required facilities and equipment. The mode of piggyback transportation and reserved train carriage is based on passenger train timetable, so it is assumed that it can meet the timeliness needs of different transportation products. The high-speed rail express train does not conflict with the passenger train.

6.2. Model Formulation. In the distribution stage, it is important to consider the relationship between transportation demand, transportation products, and service modes to ensure that the organizational model can effectively support transportation supply, and ultimately ensure that transportation supply can meet transportation demand. The logical relationship transformed into mathematics can be expressed as the expression of the variable $y_{\omega, m}^{n}$ related to the transportation organization mode and the variable $x_{\omega}^{n}$ related to the service mode stop.

6.2.1. Objective Function. The objective function of the assignment problem is to maximize the revenue of highspeed rail.

$$
\begin{aligned}
\max Z_{3}= & \sum_{n} \sum_{\omega} c_{\omega}^{n} \cdot Q_{\omega}^{n}-\sum_{i \in F} f_{i}^{m} \\
& \times\left(c_{i}^{F}+\sum_{(j, k) \in \mathcal{E}} c_{i}^{V} \cdot z_{j k} \cdot d_{j k}+\sum_{s \in \phi} x_{i}^{s} \cdot c_{i}^{t}\right) .
\end{aligned}
$$

$c_{\omega}^{n}$ is the unit cost of a certain transportation product. $Q_{\omega}^{n}$ is the demand of OD for the $n$-th transport product, $f_{i}^{m}$ represents the operation frequency of mode $m$.

\subsubsection{Constraints}

(1) Demand satisfaction constraints

$$
\sum_{n} \sum_{m} y_{\omega, m}^{n} \cdot q_{i, m}^{\omega} \geq \sum_{m} Q_{\omega}^{m}, \quad \forall \omega \in \Omega
$$


where $y_{\omega, m}^{n}$ is a $0-1$ variable, and 1 means that the demands of $\omega$ is completed by the $m$-th organization mode of the $n$-th transportation product, otherwise it is $0 . q_{i, m}^{\omega}$ refers to the loading capacity of the $i$ th train serving the $\omega$ applying the $m$-th organization mode. This constraint ensures that the railway organization scheme can meet the needs of different transportation products between OD.

(2) Capacity constraints

$$
\sum_{i \in F} f_{i} \times \overline{z_{j, k}^{l}} \leq C_{r, s}, \quad \forall(j, k) \in \varepsilon
$$

For the whole freight train, there must be a fixed capacity for freight trains in different sections of the high-speed railway network. The constraint means that for each section $(j, k)$, the total number of trains passing through the section is not greater than the upper limit of the freight train capacity in the section.

(3) Coupling constraints of freight flow and operation scheme

$$
q_{\omega}^{i} \leq \overline{y_{\omega}^{l}} \times f_{i} \times M, \quad \forall i \in F, \omega \in \Omega .
$$

The constraint means that for a train passing through an OD, if train $i$ cannot serve $\omega$, then $y_{\omega}^{i}=0$, then the flow of OD loaded by train $i$ is 0 . If train $i$ serves the od pair, then $y_{\omega}^{i}=1$.

(4) Product supply characteristics constraints

$$
\begin{aligned}
x_{\omega}^{m} & =0, \quad \forall \omega \in \Omega, m=1, \\
\sum_{m} \eta \cdot V_{i}^{m} \cdot f_{i}^{m} & \leq Q_{\omega, m}^{i}, \quad \forall i \in T, \forall \omega \in \Omega .
\end{aligned}
$$

Among them, $\eta$ is the full load rate of train. Considering the characteristics of product supply, $m=1$ means that the service mode is confirmed train, and $m=4$ means that the service mode is high-speed rail freight train. As the confirmed train running time is after $4 \mathrm{am}$, it cannot meet the demand of the product collection on that day, so the application of confirmed train mode cannot serve the product on that day. In addition, in order to ensure the revenue of high-speed rail express train, the minimum loading rate of high-speed rail express train should be met.

(5) Frequency constraints

$$
f_{i, m} \in N, \quad \forall i \in T, m=4 .
$$

The constraint ensures that the train frequency is an integer.

\section{Experimental Case Study}

7.1. Parameter Value. Beijing-Guangzhou high-speed railway is an important passenger transport channel connecting Beijing and Guangzhou, with a total length of $2298 \mathrm{~km}$. It is one of the longest high-speed railway lines in China. BeijingGuangzhou high-speed railway passes through six provinces and cities, including Beijing, Hebei, Henan, Hubei, Hunan, and Guangdong, connecting the important national economic regions, with huge freight demand.

7.1.1. Parameter Calibration. In order to calibrate $\theta_{1 n}, \theta_{2 n}$, and $\theta_{3 n}$, the process of $N$ customers selecting $m$ kinds of transportation products is regarded as $N$ times Bernoulli test, and the maximum likelihood estimation method is used to obtain the result. Through the statistical analysis of a three-month high-speed rail freight flow and customer survey data of high-speed rail express operation company, the values of relevant parameters can be obtained, as shown in Table 2.

It can be regarded as a maximum likelihood estimation function in the case of discrete distribution. After taking the logarithm on both sides of equation (14), the partial derivatives of $\theta_{1 n}, \theta_{2 n}$, and $\theta_{3 n}$ are calculated, respectively, and their derivatives are made 0 .

$$
\frac{\partial \operatorname{Inf}}{\partial \theta_{m}}=\sum_{i=1}^{m} \frac{\exp \left(V_{\text {in }} / \bar{V}\right) \cdot\left(\partial V_{\text {in }} / \partial \theta_{m}\right)-\exp \left(V_{\text {in }} / \bar{V}\right) \cdot\left[\sum_{i=1}^{m} \exp \left(V_{\text {in }} / \bar{V}\right) \cdot\left(\partial V_{\text {in }} / \partial \theta_{\text {in }}\right)\right]}{\left[\sum_{i=1}^{m} \exp \left(V_{\text {in }} / \bar{V}\right)\right]^{2}}=0 .
$$

Through MATLAB programming, the parameters are obtained.

$$
\theta_{\text {in }}=\left[\begin{array}{lll}
0.1263 & 0.6966 & 0.4630 \\
0.0927 & 0.1585 & 0.0138 \\
0.1082 & 0.030 & 0.0069
\end{array}\right] \text {. }
$$

Substituting equations (14)-(16), the freight flow sharing rate of the freight product based on customers' choice intention is obtained, and the results are shown in Table 3.
7.1.2. Modified Calculation of Freight Flow Share Rate of High-Speed Rail Freight Products. In actual operation, the railway will give priority to the loading of goods arriving on the Same-day delivery, followed by the Next Morning Delivery, the Next Day Service, and the Third-day delivery, respectively. Therefore, considering the characteristics of transportation organization, and comparing the freight flow share rate of various freight products and the operation proportion of various products, the Same-day delivery and the Next Morning Delivery cannot effectively meet the needs of customers, and this part of freight flow will be transferred. The probability matrix of freight flow transfer is shown below: 
TABLE 2: Statistical results of various types of freight flow.

\begin{tabular}{lccccc}
\hline & $D$ & $C$ & $r$ & $s$ & \\
\hline Freight flow 1 & 827 & 202 & 6 & 0 & 12.18 \\
Freight flow 2 & 256 & 2361 & 2415 & 894 & 69.74 \\
Freight flow 3 & 0 & 83 & 520 & 933 & 18.08 \\
\hline
\end{tabular}

TABLe 3: Freight flow sharing rate calculation results of the two stages.

\begin{tabular}{|c|c|c|c|c|}
\hline High-speed rail products & $d$ & $C$ & $r$ & $s$ \\
\hline Based on customer choice intention (\%) & 17.91 & 32.92 & 29.43 & 19.74 \\
\hline Proportion of freight flow transfer (\%) & -8.93 & 5.47 & 3.46 & - \\
\hline Revised freight flow share rate (\%) & 8.98 & 38.39 & 32.89 & 19.74 \\
\hline Actual share rate $(\%)$ & 9.73 & 36.42 & 36.59 & 17.26 \\
\hline
\end{tabular}

$$
P=\left[\begin{array}{lll}
\alpha_{d \longrightarrow c} & \alpha_{d \longrightarrow r} & \alpha_{d \longrightarrow s} \\
\alpha_{c \longrightarrow d} & \alpha_{c \longrightarrow r} & \alpha_{c \longrightarrow s} \\
\alpha_{r \longrightarrow d} & \alpha_{r \longrightarrow c} & \alpha_{r \longrightarrow s} \\
\alpha_{s \longrightarrow d} & \alpha_{s \longrightarrow c} & \alpha_{s \longrightarrow r}
\end{array}\right]=\left[\begin{array}{ccc}
0.8534 & 0.1466 & 0 \\
0 & 0.1955 & 0 \\
0 & 0 & 0 \\
0 & 0 & 0
\end{array}\right]
$$

According to the abovementioned calculation method considering freight flow transfer, the revised freight flow share rate can be obtained. The revised value, the revised freight flow share rate, and the actual share rate of Beijing-Guangzhou high-speed railway are shown in Table 3.

It can be seen from Table 3 that the freight flow sharing rate of the freight product after considering the freight flow transfer is more in line with the actual situation, which indicates the feasibility of the calculation method of freight flow sharing rate of high-speed rail constructed in this paper, and provides theoretical support for further reasonable allocation of transport capacity resources.

After defining the requirements of each product, it is necessary to further determine the relevant parameters of the transportation organization scheme. Express trains of highspeed railway have the same speed level, and the difference of train types lies in the different train stops, which can be divided into multi station stop and through train. The train stop mode shall be flexibly arranged according to the OD of goods source. At present, the carrying capacity of the highspeed rail express train is 87 tons. According to the forecast of high-speed rail express demand in 2025, the average daily OD volume of high-speed rail express between Beijing and Guangzhou is shown in Table 4.

The fixed $\operatorname{cost} c_{i}^{F}$ for a single train is 420 thousand CNY. In addition to the fixed cost, variable cost is divided into the following:

(1) Train running variable cost $c_{i}^{V} 700 \mathrm{CNY} / \mathrm{km}$, which is related to distance.

(2) Variable cost $c_{i}^{S}$ takes 50 thousand CNY/times, which is related to the number of the times the train stops.

(3) The variable cost $c^{L}$ of loading and unloading the goods at the site is $10 \mathrm{CNY} /$ ton.
It is assumed that the passing capacity $C_{\mathrm{rs}}$ of each section can meet the requirements of high-speed rail express train operation.

The distance between cities is shown in Table 5 .

7.2. Service Path Generation. In this paper, we used the python language to invoke the CPLEX12.7.1 engine to realize the column generation algorithm. The calculations are made with a laptop computer. Due to the small scale of the case, the computational time took only 1 second.

7.2.1. The Train Candidate Set Scheme in the down Direction. As can be seen from Figure 3, there are 10 types of trains that can operate in the down direction. From the perspective of train type and quantity, as the fixed cost of single train operation is far greater than the cost of stopping, there are 8 trains with at least one stop, and only 3 through trains. Among them, there are 6 long-distance trains between Beijing and Guangzhou, which effectively apportions the cost of single train operation. From the point of departure, stop, and destination, there are 9 trains from Beijing, which is in line with the prediction of Beijing as an important source of goods. Zhengzhou and Wuhan start one train each to meet the demand of express goods.

7.2.2. The Train Candidate Set Scheme in the up Direction. As can be seen from Figure 4, there are 10 types of trains in the service path of up going trains, which can effectively meet the demand of freight transportation between cities.

7.3. Freight Flow Allocation Scheme. Furthermore, the transportation demand and train alternative set are input into the freight flow allocation model, and the decisionmaking scheme of high-speed rail product transportation mode between Beijing and Guangzhou main nodes is obtained. Because there are many combinations of OD demand and transportation products, only some decision schemes are given, as shown in Table 6.

It can be seen from Table 6 that the four transportation products match the four organization modes, realizing the 
TABLE 4: High-speed rail express demand of main nodes between Beijing and Guangzhou $(t)$.

\begin{tabular}{|c|c|c|c|c|c|c|}
\hline \multirow{2}{*}{$O$} & \multicolumn{6}{|c|}{$D$} \\
\hline & Beijing & Shijiangzhuang & Zhengzhou & Wuhan & Changsha & Guangzhou \\
\hline Beijing & - & 61.4 & 127.5 & 69.9 & 45.1 & 134.0 \\
\hline Shijiazhuang & 62.2 & - & 17.4 & 13.0 & 7.0 & 15.0 \\
\hline Zhengzhou & 102.3 & 15.8 & - & 30.5 & 17.2 & 32.2 \\
\hline Wuhan & 48.3 & 11.3 & 29.4 & - & 24.7 & 56.5 \\
\hline Changsha & 22.7 & 4.5 & 12.1 & 18.0 & - & 46.0 \\
\hline Guangzhou & 264.5 & 53.6 & 127.7 & 232.5 & 259.5 & - \\
\hline
\end{tabular}

TABLe 5: The distance between major cities $(\mathrm{km})$.

\begin{tabular}{|c|c|c|c|c|c|c|}
\hline City & Beijing & Shijiangzhuang & Zhengzhou & Wuhan & Changsha & Guangzhou \\
\hline Beijing & - & 265 & 639 & 1102 & 1397 & 1967 \\
\hline Shijiazhuang & 265 & - & 374 & 837 & 1132 & 1702 \\
\hline Zhengzhou & 639 & 374 & - & 463 & 758 & 1328 \\
\hline Wuhan & 1102 & 837 & 463 & - & 295 & 865 \\
\hline Changsha & 1397 & 1132 & 758 & 295 & - & 570 \\
\hline Guangzhou & 1967 & 1702 & 1328 & 865 & 570 & - \\
\hline
\end{tabular}

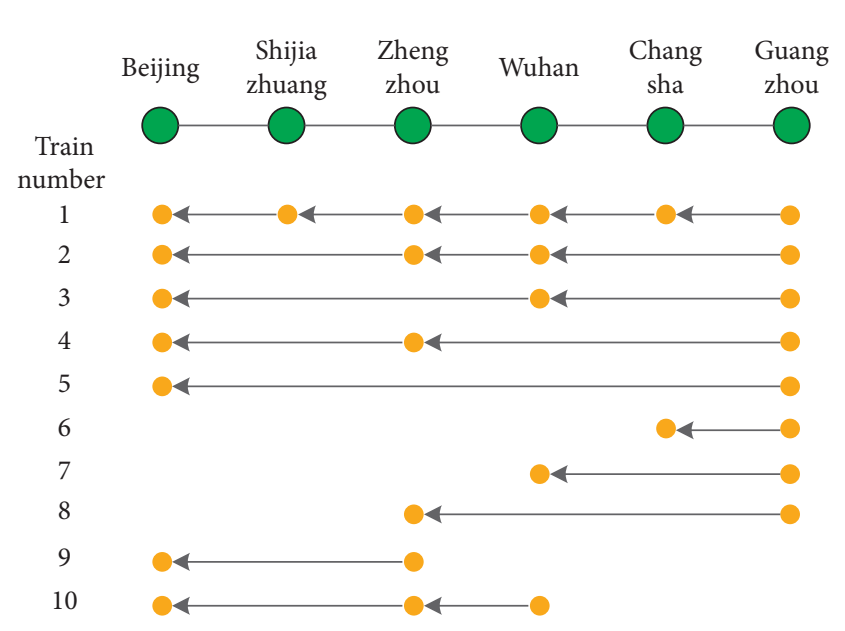

Figure 4: The backup plan for high-speed rail express trains in the up direction.

TABLE 6: Decision-making scheme of high-speed rail express product transportation mode.

\begin{tabular}{|c|c|c|c|c|c|c|}
\hline \multirow{2}{*}{ OD } & \multirow{2}{*}{ Product } & \multirow{2}{*}{ Transportation demand $(t)$} & \multicolumn{4}{|c|}{ Share of transport modes $(t)$} \\
\hline & & & $m=1$ & $m=2$ & $m=3$ & $m=4$ \\
\hline \multirow{3}{*}{ Beijing-Shijiazhuang } & $n=1$ & 6.11 & 0 & 6.11 & 0 & 0 \\
\hline & $n=2$ & 28.23 & 0 & 28.23 & 0 & 0 \\
\hline & $n=3$ & 33.19 & 6.03 & 0 & 0 & 27.16 \\
\hline \multirow{4}{*}{ Beijing-Zhengzhou } & $n=1$ & 12.71 & 0 & 12.71 & 0 & 0 \\
\hline & $n=2$ & 51.74 & 2.99 & 13.32 & 0 & 35.43 \\
\hline & $n=3$ & 52.38 & 0 & 0 & 14.06 & 38.28 \\
\hline & $n=4$ & 29.20 & 3.19 & 0 & 0 & 26.01 \\
\hline \multirow{4}{*}{ Beijing-Wuhan } & $n=1$ & 3.05 & 0 & 3.05 & 0 & 0 \\
\hline & $n=2$ & 22.53 & 0 & 6.24 & 7.20 & 9.09 \\
\hline & $n=3$ & 15.76 & 4.01 & 0 & 0 & 11.75 \\
\hline & $n=4$ & 10.66 & 0 & 0 & 0 & 10.66 \\
\hline \multirow{4}{*}{ Beijing-Changsha } & $n=1$ & 9.06 & 2.17 & 2.59 & 4.3 & 0 \\
\hline & $n=2$ & 49.28 & 0 & 9.28 & 12.08 & 28.92 \\
\hline & $n=3$ & 55.37 & 4.03 & 2.21 & 21.58 & 27.55 \\
\hline & $n=4$ & 22.64 & 2.96 & 0 & 0 & 19.68 \\
\hline \multirow{4}{*}{ Beijing-Guangzhou } & $n=1$ & 14.37 & 4.17 & 2.20 & 8 & 0 \\
\hline & $n=2$ & 61.42 & 0 & 9.28 & 12.08 & 40.06 \\
\hline & $n=3$ & 52.62 & 2.89 & 1.09 & 19.6 & 29.04 \\
\hline & $n=4$ & 31.58 & 3.06 & 0 & 0 & 28.52 \\
\hline
\end{tabular}




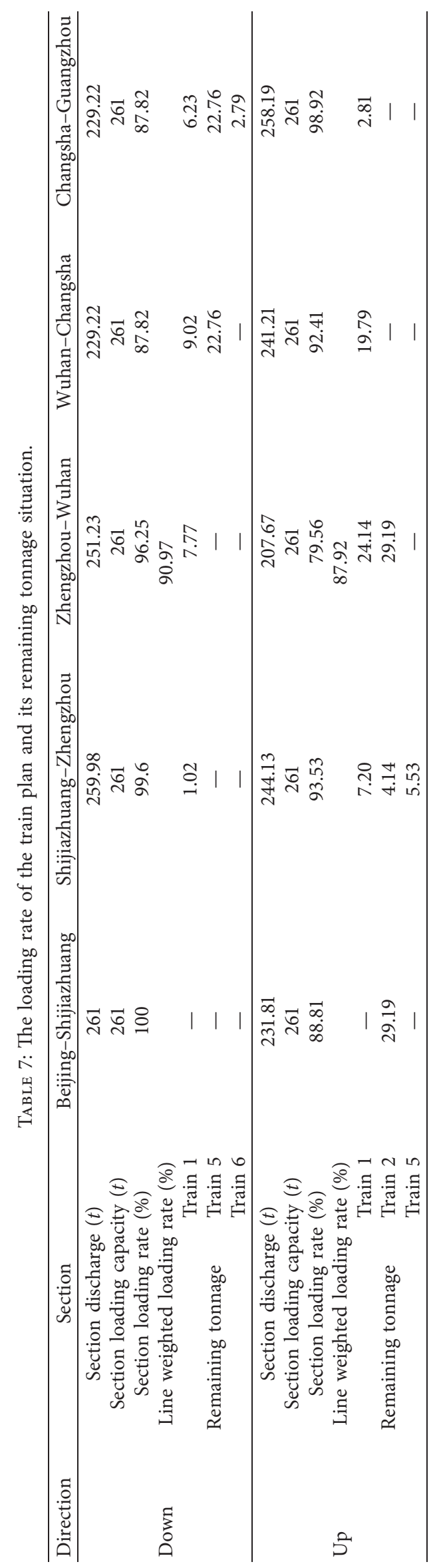


accurate distribution of freight flow. From the perspective of different transportation organization modes, it gives full play to the flexible characteristics of small batch and multi batch of confirmation car and piggyback transportation, undertakes the freight demand of part of short distance, and the reserved car and high-speed rail freight train mode undertakes the main transportation tasks in medium and long distance, giving full play to the advantages of running between large nodes.

In order to further analyze the loading efficiency of freight multiple units and the utilization of sections, the section flow of each section and the remaining carrying capacity of specific trains are shown in Table 7.

In order to show the advantages of the two-stage model constructed in this paper, the first stage of intelligent generation of service path is replaced by the full enumeration method to generate the alternative set and the alternative set given by artificial experience judgment, which are, respectively, used as the input conditions of the second stage of assignment model to solve. The results show that:

(1) When allocating the candidate set generated by the full enumeration method, the efficiency of the solution is changed from 2 seconds to 13 minutes due to the larger scale of the problem, and the allocation scheme is consistent with the two-stage model mentioned above.

(2) When allocating the alternative set judged by manual experience, due to the inability to accurately judge the reasonable alternative set, the freight flow cannot be allocated to the optimal service path. As a result, in some schemes, due to the inability to meet the demand of freight flow, four additional trains are opened, which increases the fixed cost compared with the above scheme. In some schemes, because there is no suitable service path, the train loading rate is poor.

\section{Conclusions}

High-speed rail freight is an important means to effectively meet the high-end logistics market, and the market demand will increase significantly year by year. The freight demand calculation method designed in this paper effectively considers the matching relationship between supply and demand. At the same time, the demand-based organization scheme design effectively balances the relationship between cost and benefit. In the aspect of product demand calculation, we consider the freight flow calculation method based on customers' choice intention and the freight flow correction method based on actual supply capacity, which effectively distinguish the influence of different transportation products and supply on transportation organization mode. In the next calculation, we optimize the service path model, and focus on improving the second stage cargo flow allocation model to ensure that the transportation supply can effectively meet the needs of different transportation products. The research results show that the freight flow sharing rate calculation method designed by us can better reflect the actual freight demand, and the organization scheme can effectively meet the needs of different transportation products, and the calculation results are very encouraging.

At present, when we revise the product freight flow share rate, we revise it based on the existing effective supply, without considering the different income of different transportation products. In the future, we can further consider the income of different products and strategically choose the transportation supply mode to improve the overall efficiency of high-speed rail freight.

\section{Data Availability}

The data used to support the findings of this study are available from the corresponding author upon request.

\section{Conflicts of Interest}

The authors declare no conflicts of interest with respect to the research, authorship, and publication of this article.

\section{Acknowledgments}

Supported by National Key R\&D Program of China (Grant No. 2018YFB1601303) and Scientific research projects of China Academy of Railway Sciences Group Co., Ltd (Grant No. 2020YJ196).

\section{References}

[1] X. Yu, M. Lang, Y. Gao et al., "An empirical study on the design of China high-speed rail express train operation planfrom a sustainable transport perspective," Sustainability, vol. 10, no. 7, p. 2478, 2018.

[2] X. Yu, M. Lang, W. Zhang, S. Li, M. Zhang, and X. Yu, “An empirical study on the comprehensive optimization method of a train diagram of the China high speed railway express," Sustainability, vol. 11, no. 7, p. 2141, 2019.

[3] A. Talebian and B. Zou, "Integrated modeling of high performance passenger and freight train planning on shared-use corridors in the US," Transportation Research Part B: Methodological, vol. 82, pp. 114-140, 2015.

[4] M. P. Socorro and M. F. Viecens, "The effects of airline and high speed train integration," Transportation Research Part A: Policy and Practice, vol. 49, no. 49, pp. 160-177, 2013.

[5] K. Arunotayanun and J. W. Polak, "Taste heterogeneity and market segmentation in freight shippers' mode choice behaviour," Transportation Research Part E: Logistics and Transportation Review, vol. 47, no. 2, pp. 138-148, 2011.

[6] R. Danielis, E. Marcucci, and L. Rotaris, "Logistics managers' stated preferences for freight service attributes," Transportation Research Part E: Logistics and Transportation Review, vol. 41, no. 3, pp. 201-215, 2005.

[7] H. Sonntag, "Ein verfahren zur alternativroutensuche in verkehrsnetzen," Computing, vol. 21, no. 4, pp. 323-331, 1979.

[8] M. Carey, "A model and strategy for train pathing with choice of lines, platforms, and routes," Transportation Research, Part B (Methodological), vol. 28, no. 5, pp. 350-353, 1994. 
[9] A. Caprara, M. Fischetti, and P. Toth, "Modeling and solving the train timetabling problem," Operations Research, vol. 50, no. 5, pp. 851-861, 2002.

[10] B. A. A. Assad, "Modelling of rail networks: towards a routing/makeup model," Transportation Research, vol. 14, no. $1,1980$.

[11] M. T. Claessens, N. M. van Dijk, and P. J. Zwaneveld, "Cost optimal allocation of rail passenger lines," European Journal of Operational Research, vol. 110, no. 3, pp. 474-489, 1998.

[12] I. Racunica and L. Wynter, "Optimal location of intermodal freight hubs," Transportation Research Part B, vol. 39, no. 5, pp. 453-477, 2000.

[13] M. A. Ertem and M. Keskin Özcan, "Freight transportation using high-speed train systems," Transportation Letters, vol. 8, no. 5, pp. 250-258, 2016.

[14] T. Lindner and U. T. Zimmermann, "Cost optimal periodic train scheduling," Mathematical Methods of Operations Research, vol. 62, no. 2, pp. 281-295, 2005.

[15] J.-W. Goossens, S. Van Hoesel, and L. Kroon, "A branch-andcut approach for solving railway line-planning problems," Transportation Science, vol. 38, no. 3, pp. 379-393, 2004.

[16] M. R. Bussieck, T. Lindner, and M. E. Lübbecke, "A fast algorithm for near cost optimal line plans," Mathematical Methods of Operations Research (ZOR), vol. 59, no. 2, pp. 205-220, 2004.

[17] A. Schöbel, Line Planning in Public Transportation: Models and Methods, Springer-Verlag New York, Inc, New York, NY, USA, 2012.

[18] F. Jiang, V. Cacchiani, and P. Toth, "Train timetabling by skipstop planning in highly congested lines," Transportation Research Part B: Methodological, vol. 104, pp. 149-174, 2017.

[19] S. Burggraeve and P. Vansteenwegen, "Robust routing and timetabling in complex railway stations," Transportation Research Part B: Methodological, vol. 101, pp. 228-244, 2017.

[20] V. Cacchiani, A. Caprara, and P. Toth, "A column generation approach to train timetabling on a corridor," $4 O R$, vol. 6 , no. 2, pp. 125-142, 2008.

[21] J. Qi, L. Yang, Z. Di, S. Li, K. Yang, and Y. Gao, "Integrated optimization for train operation zone and stop plan with passenger distributions," Transportation Research Part E: Logistics and Transportation Review, vol. 109, pp. 151-173, 2018.

[22] Y. Wang, Study on High-Speed Railway Network Line Planning for Extra-long Distance Passenger Flow, Beijing Jiaotong University, Beijing, China, 2018.

[23] Y.-L. Wang, T. Zhou, J.-J. Shi, J. Wang, and D.-R. He, "Empirical analysis of dependence between stations in Chinese railway network," Physica A: Statistical Mechanics and Its Applications, vol. 388, no. 14, pp. 2949-2955, 2009.

[24] S. C. Wong, C. Yang, and H. K. Lo, "A path-based traffic assignment algorithm based on the TRANSYT traffic model," Transportation Research Part B: Methodological, vol. 35, no. 2, pp. 163-181, 2001.

[25] M. E. Pfetsch and R. Borndrfer, "Routing in line planning for public transport," in Proceedings of the Operations Research Proceedings, Bremen, Germany, September 2006.

[26] D. Bertsimas and M. Sim, "The price of robustness," Operations Research, vol. 45, no. 2, pp. 161-162, 2005.

[27] L. M. Torres, R. Torres, R. Borndörfer, and M. E. Pfetsch, "Line planning on tree networks with applications to the Quito Trolebús system," International Transactions in Operational Research, vol. 18, no. 4, pp. 455-472, 2011. 